\title{
Harmonic and sub-harmonic resonance of MEMS subjected to a weakly non-linear parametric and external excitations
}

\author{
A. M. Elnaggar, A. F. El-Bassiouny and G. A. Mosa* \\ Department of Mathematics,Faculty of Science, Benha University, Egypt, B. O. 13518 \\ *Corresponding author E-mail: gamal_graph@yahoo.com
}

\begin{abstract}
This paper presents a simplified mathematical model for the purpose of studying the resonant responses of a nonlinear dynamical system, (micro - electro - mechanical systems (MEMS)), which represented by a Van-der Pol equation subjected to a weakly non-linear parametric and forcing excitations. Using Multiple scales method, the Van-der Pol equation is transformed to a system of second order differential equation up to first order of small parameter $\varepsilon$. Three types of resonances are studied (harmonic resonance and subharmonic resonances of even order (one half and one - fourth )). The modulation equations for each resonances, steady state solutions, frequency-response equations, stability analysis are determined. Numerical analysis for frequency-response equations and stability conditions are carried out. Results are presented graphically by group of figures. Finally discussion for these figures are given.
\end{abstract}

Keywords: Micro-cantilever, Parametric and Forcing excitations, Resonances and Stability, Weakly non-linear dynamical systems.

\section{Introduction}

Nonlinearities in micro-electro-mechanical systems (MEMS) can arise from various sources such as spring and damping mechanisms [1,2] and resistive, inductive, and capacitive circuit elements [3]. Surface and fluids forces $[4,5]$ can also be other sources of nonlinearity. However, the inherent source of nonlinearity in electrostatic MEMS is the nonlinear coupling between the electrostatic force and the displacement of the MEM structure [6]. This nonlinearity has been shown to give rise to various dynamical behavior like Harmonic and Sub-harmonic resonances response. There are various methods can be used to drive MEM structures at resonance. The most commonly used method is the primary-resonance excitation, in which the frequency of the excitation is tuned closed to the fundamental natural frequency of the microstructure. Examples of this excitation in MEMS can be found in various applications, such as resonant sensors [7] and RF filters [8]. Jin and Wang [9] showed that driving a resonant microsensor with a subharmonic excitation of order one-half increased the signal-to-crosstalk ratio as compared to driving it at primary resonance. Younis et al. $[7,10]$ used the method of multiple scales to study the response of an electrostatically actuated resonator to a primary resonance excitation. Also Abdel-Rahman and Nayfeh [11] studied a superharmonic resonance excitation of order two, and a subharmonic resonance excitation of order one-half. Furthermore, Nayfeh and Younis [12] investigated the dynamics of a MEMS resonator to a subharmonic resonance of order one-half and to a superharmonic resonance of order two. Kacem et al. [13] studied the nonlinear dynamics of nanomechanical beam resonators to improve the performance of MEMS-based sensors. Alsaleem et al. [14] investigated the nonlinear phenomena, including primary resonance, superharmonic and subharmonic resonances, in electrostatically actuated resonators both experimentally and theoretically. Zhang and Meng [15] analyzed the nonlinear dynamics of the electrostatically actuated resonant MEMS sensors under parametric excitation. The problem of studied the response "Harmonic, Sub-harmonic and super-harmonic" of weakly non-linear dynamical system subjected to external excitation, parametric excitation or both is investigated by [16, 17].

In this paper harmonic resonance and subharmonic resonances of order(one - half and one - fourth ) are investigated by applying the method of multiple scales. For each type of resonance we determine steady-state solution, 
frequency response equation and stability of the steady-state solution. Frequency response curves are plotted in which solid curve represent stable solutions and dashed curve represent unstable solutions. Finally discussion for the figures are given.

\section{Formulation of the problem and perturbation analysis}

The governing equation of motion for a simplified dynamic system of the micro-cantilever beam in MEMS [18] is represented by the following nonlinear second order differential equation

$$
\ddot{u}+\Psi(u, \dot{u})+\omega_{0}^{2} u+f_{0}(u)+q(t) \cdot e(u)=p(t)
$$

where

$\Psi(u, \dot{u})=2 \epsilon\left(\mu_{0} \dot{u}+\mu_{1} u^{2} \dot{u}\right), f_{0}(u)=0, q(t) \cdot e(u)=\epsilon\left(\alpha_{1} u+\alpha_{2} u^{2}+\alpha_{3} u^{3}+\alpha_{4} u^{4}\right) \cos (\Omega t)$ and $p(t)=\epsilon F \cos (\Omega t)$. then, equation (1) becomes

$$
\ddot{u}+\omega_{0}^{2} u+2 \epsilon\left(\mu_{0} \dot{u}+\mu_{1} u^{2} \dot{u}\right)+\epsilon\left(\alpha_{1} u+\alpha_{2} u^{2}+\alpha_{3} u^{3}+\alpha_{4} u^{4}\right) \cos (\Omega t)=\epsilon F \cos (\Omega t)
$$

Equation (2) represent a Van-der Pol equation subjected to weakly non-linear parametric and forcing excitations, where, $\mathrm{F}$ is the amplitude of forcing excitation, $\Omega$ is the frequency of parametric and external excitations, $\mu_{0}$ and $\mu_{1}$ are the coefficients of viscous linear and nonlinear damping terms and $\alpha_{n}(n=1,2,3,4)$ are the coefficients of nonlinear parametric excitation.

Using the method of Multiple Scales (Nayfeh [19]), we get a first order uniform solution of equation (2) in the form

$$
u(t ; \epsilon)=u_{0}\left(T_{0}, T_{1}\right)+\epsilon u_{1}\left(T_{0}, T_{1}\right)+\ldots,
$$

where $T_{0}=t$ is the first scale associated with changes occurring at the frequencies $\omega_{0}$ and $\Omega$, and $T_{1}=\epsilon t$ is a slow scale associated with modulations in the amplitude. In terms of $T_{1}$, the time derivatives become

$$
\frac{d}{d t}=D_{0}+\epsilon D_{1}+\ldots \quad \quad \quad \frac{d^{2}}{d t^{2}}=D_{0}^{2}+2 \epsilon D_{0} D_{1}+\ldots
$$

where $D_{n}=\frac{\partial}{\partial T_{n}}$. Substituting equations (3) and (4) into equation (2) and equating coefficients of like powers of $\epsilon$ one obtains

$$
\begin{aligned}
& D_{0}^{2} u_{0}+\omega_{0}^{2} u_{0}=0 \\
& \left.D_{0}^{2} u_{1}+\omega_{0}^{2} u_{1}=-2\left(\mu_{0} D_{0} u_{0}+\mu_{1} u_{0}^{2} D_{0} u_{0}+D_{0} D_{1} u_{0}\right)+\left(F-\alpha_{1} u_{0}-\alpha_{2} u_{0}^{2}-\alpha_{3} u_{0}^{3}-\alpha_{4} u_{0}^{4}\right) \cos \left(T_{0} \Omega\right)\right)
\end{aligned}
$$

The solution of equation (5)can be expressed in the complex form

$$
u_{0}=A e^{i \omega_{0} T_{0}}+\bar{A} e^{-i \omega_{0} T_{0}}
$$

where $\bar{A}$ is the complex conjugate of $A$. Then equation (6) becomes,

$$
\begin{aligned}
D_{0}{ }^{2} u_{1}+\omega_{0}^{2} u_{1}= & -2 i \omega_{0}\left(\mu_{0} A+\mu_{1} A^{2} \bar{A}+A^{\prime}\right) e^{i \omega_{0} T_{0}} \\
& +\left(\frac{1}{2} F-\alpha_{2} A \bar{A}-3 \alpha_{4} A^{2} \bar{A}^{2}\right) e^{i \Omega T_{0}} \\
& -\frac{1}{2}\left(\alpha_{1} \bar{A}+3 \alpha_{3} A \bar{A}^{2}\right) e^{i\left(\Omega-\omega_{0}\right) T_{0}} \\
& -\left(\frac{1}{2} \alpha_{2} \bar{A}^{2}+2 \alpha_{4} A \bar{A}^{3}\right) e^{i\left(\Omega-2 \omega_{0}\right) T_{0}} \\
& -\frac{1}{2} \alpha_{3} \bar{A}^{3} e^{i\left(\Omega-3 \omega_{0}\right) T_{0}} \\
& -\frac{1}{2} \alpha_{4} \bar{A}^{4} e^{i\left(\Omega-4 \omega_{0}\right) T_{0}} \\
& +N S T .+ \text { c.c. }
\end{aligned}
$$

where $N S T$. denotes the terms does not produce secular terms and c.c. denotes the complex conjugate.

Equation (8) contain five cases of resonance

$$
\Omega \simeq n \omega_{0} \quad ; n=1,2,3,4,5
$$

In this paper we restricted our attention to three cases of resonance (harmonic resonance and subharmonic resonances of order(one - half and one - fourth )). 


\section{$3 \quad$ Harmonic (primary) resonance $\left(\Omega \simeq \omega_{0}\right)$}

To describe the nearness of the excitation frequency $\Omega$ to the fundamental natural frequency $\omega_{0}$ i.e. $\Omega \simeq \omega_{0}$, we introduce the detuning parameter $\sigma$ to convert the small-divisor terms into secular terms according to

$$
\Omega=\omega_{0}+\epsilon \sigma
$$

where $\sigma$ is the detuning parameter. Eliminating the secular terms from the equation (8) yields

$$
-2 i \omega_{0}\left(\mu_{0} A+\mu_{1} A^{2} \bar{A}+A^{\prime}\right)+\left(\frac{1}{2} F-\alpha_{2} A \bar{A}-3 \alpha_{4} A^{2} \bar{A}^{2}\right) e^{i \sigma T_{1}}=0
$$

where the prime (') indicates to the derivative with respect to $T_{1}$. Writing $A$ in the polar form as $A=$ $\frac{1}{2} a\left(T_{1}\right) e^{i \beta\left(T_{1}\right)}$ into equation (10) where $a\left(T_{1}\right)$ and $\beta\left(T_{1}\right)$ are real-valued functions, representing, respectively, the amplitude and phase of the response. Separating real and imaginary parts, we obtain the following modulation equations:

$$
\begin{aligned}
& a^{\prime}=\frac{1}{2 \omega_{0}}\left(F-\frac{1}{2} \alpha_{2} a^{2}-\frac{3}{8} \alpha_{4} a^{4}\right) \sin (\gamma)-\mu_{0} a-\frac{1}{4} \mu_{1} a^{3} \\
& a \gamma^{\prime}=a \sigma+\frac{1}{2 \omega_{0}}\left(F-\frac{1}{2} \alpha_{2} a^{2}-\frac{3}{8} \alpha_{4} a^{4}\right) \cos (\gamma)
\end{aligned}
$$

where $\gamma=\sigma T_{1}-\beta\left(T_{1}\right)$.

For steady state solution, $a^{\prime}=\gamma^{\prime}=0$, in equations (11) and (12) we obtain

$$
\begin{aligned}
& a \omega_{0}\left(\mu_{0}+\frac{1}{4} \mu_{1} a^{2}\right)=\frac{1}{2}\left(F-\frac{1}{2} \alpha_{2} a^{2}-\frac{3}{8} \alpha_{4} a^{4}\right) \sin (\gamma) \\
& a \omega_{0} \sigma=-\frac{1}{2}\left(F-\frac{1}{2} \alpha_{2} a^{2}-\frac{3}{8} \alpha_{4} a^{4}\right) \cos (\gamma)
\end{aligned}
$$

Equations (13) and (14) show that there are no trivial solution at $a=0$. For non-trivial solution i.e. at $a \neq 0$, eliminating $\gamma$ from equations (13) and (14), we get the following frequency-response equation

$$
a^{2}\left[\sigma^{2}+\left(\mu_{0}+\frac{1}{4} \mu_{1} a^{2}\right)^{2}\right]=\frac{1}{4 \omega_{0}^{2}}\left(F-\frac{1}{2} \alpha_{2} a^{2}-\frac{3}{8} \alpha_{4} a^{4}\right)^{2}
$$

Solving Equation (15) for $\sigma$, we obtain

$$
\sigma= \pm \sqrt{-\left(\mu_{0}+\frac{1}{4} \mu_{1} a^{2}\right)^{2}+\frac{1}{4 a^{2} \omega_{0}^{2}}\left(F-\frac{1}{2} \alpha_{2} a^{2}-\frac{3}{8} \alpha_{4} a^{4}\right)^{2}}
$$

To determined the stability of the non-trivial solutions, let

$$
a=a_{0}+a_{1}\left(T_{1}\right) \quad \& \quad \gamma=\gamma_{0}+\gamma_{1}\left(T_{1}\right)
$$

where $a_{0}$ and $\gamma_{0}$ correspond to a non-trivial solutions and $a_{1}$ and $\gamma_{1}$ are perturbations which are assumed to be small compared with $a_{0}$ and $\gamma_{0}$. Substituting (17) into equations (11) and (12) and linearizing the resulting equations, we obtain

$$
\begin{aligned}
& a_{1}^{\prime}=-\frac{a_{0}^{2} \mu_{1}\left(-3 a_{0}^{4} \alpha_{4}+4 a_{0}^{2} \alpha_{2}-24 F\right)-4 \mu_{0}\left(9 a_{0}^{4} \alpha_{4}+4 a_{0}^{2} \alpha_{2}+8 F\right)}{4\left(3 a_{0}^{4} \alpha_{4}+4 a_{0}^{2} \alpha_{2}-8 F\right)} a_{1}-a_{0} \sigma \gamma_{1} \\
& \gamma_{1}^{\prime}=\frac{\sigma\left(9 a_{0}^{4} \alpha_{4}+4 a_{0}^{2} \alpha_{2}+8 F\right)}{-3 a_{0}^{5} \alpha_{4}-4 a_{0}^{3} \alpha_{2}+8 a_{0} F} a_{1}-\frac{1}{4}\left(a_{0}^{2} \mu_{1}+4 \mu_{0}\right) \gamma_{1}
\end{aligned}
$$


Equations (18) and (19) admit solutions of the form $\left(a_{1}, \gamma_{1}\right)=\left(\Gamma_{1}, \Gamma_{2}\right) e^{\theta T_{1}}$. The eigenvalues equation can be obtained as

$$
\theta=\frac{1}{32 g_{2}}\left(K \pm \sqrt{K^{2}+64 g_{2}\left(16 g_{1}\left(\mu_{0}^{2}+\sigma^{2}\right)+4 \mu_{0} \mu_{1}\left(g_{1}+g_{3}\right) a_{0}^{2}+g_{3} \mu_{1}^{2} a_{0}^{4}\right)}\right)
$$

where,

$g_{1}=8 F+4 \alpha_{2} a_{0}^{2}+9 \alpha_{4} a_{0}^{4}, \quad g_{2}=-8 F+4 a_{0}^{2} \alpha_{2}+3 a_{0}^{4} \alpha_{4}, \quad g_{3}=24 F-4 \alpha_{2} a_{0}^{2}+3 \alpha_{4} a_{0}^{4}$

and $K=\left(16 \mu_{0}\left(g_{1}-g_{2}\right)-4 \mu_{1}\left(g_{2}-g_{3}\right) a_{0}^{2}\right)$

Consequently, a solution is stable if and only if the real parts of both eigenvalues of (20) are less than zero.

\section{Subharmonic resonance of order $1 / 2\left(\Omega \simeq 2 \omega_{0}\right)$}

For this type of oscillations resonance, one must have $\Omega \simeq 2 \omega_{0}$, to express this nearness, let

$$
\Omega=2 \omega_{0}+\epsilon \sigma
$$

and write

$$
\left(\Omega-\omega_{0}\right) T_{0}=\omega_{0} T_{0}+\epsilon \sigma T_{0}=\omega_{0} T_{0}+\sigma T_{1}
$$

Eliminating the secular terms from the equation (8) yields

$$
2 i \omega_{0}\left(\mu_{1} A^{2} \bar{A}+A^{\prime}+\mu_{0} A\right)+\frac{1}{2}\left(3 \alpha_{3} A \bar{A}^{2}+\alpha_{1} \bar{A}\right) e^{i T_{1} \sigma}=0
$$

Using the polar form $A=\frac{1}{2} a\left(T_{1}\right) e^{i \beta\left(T_{1}\right)}$ into the equation (23) and separating real and imaginary parts, we obtain the following modulation equations:

$$
\begin{aligned}
& a^{\prime}=-\frac{1}{4}\left(\mu_{1} a^{3}+4 \mu_{0} a\right)-\frac{\left(3 \alpha_{3} a^{3}+4 \alpha_{1} a\right) \sin (\gamma)}{16 \omega_{0}} \\
& a \gamma^{\prime}=\sigma a-\frac{\left(3 \alpha_{3} a^{3}+4 \alpha_{1} a\right) \cos (\gamma)}{8 \omega_{0}}
\end{aligned}
$$

where $\gamma=\sigma \mathrm{T}_{1}-2 \beta$.

For steady state solution, $a^{\prime}=\gamma^{\prime}=0$, in equations (24) and (25) we obtain

$$
\begin{aligned}
& \left(3 \alpha_{3} a^{3}+4 \alpha_{1} a\right) \sin (\gamma)=-4 \omega_{0} a\left(\mu_{1} a^{2}+4 \mu_{0}\right) \\
& \left(3 \alpha_{3} a^{3}+4 \alpha_{1} a\right) \cos (\gamma)=8 \omega_{0} \sigma a
\end{aligned}
$$

Equations (26) and (27) show that there are two possibilities: (trivial solution ) at $a=0$ and ( nontrivial solution) at $a \neq 0$. Squaring and adding (26) and (27) we get the frequency-response equation

$$
\sigma= \pm \frac{1}{8 \omega_{0}} \sqrt{\left(3 \alpha_{3} a^{2}+4 \alpha_{1}\right)^{2}-16 \omega_{0}^{2}\left(\mu_{1} a^{2}+4 \mu_{0}\right)^{2}}
$$

The analysis of the stability of the trivial solutions is equivalent to the analysis of the linear solutions of equation (23) by neglecting the non-linear terms we get

$$
\frac{1}{2} \alpha_{1} e^{i \sigma \mathrm{T}_{1}} \bar{A}+2 i \omega_{0}\left(A^{\prime}+\mu_{0} A\right)=0
$$

to solve equation (29) one lets $A=e^{\frac{1}{2} i \sigma T_{1}}\left(B\left(T_{1}\right)+i b\left(T_{1}\right)\right)$ where B and b are real, separates real and imaginary parts and get

$$
\begin{aligned}
& \left(\frac{\alpha_{1}}{2}+\omega_{0} \sigma\right) b-2 \omega_{0} B^{\prime}-2 \omega_{0} \mu_{0} B=0 \\
& 2 \omega_{0} b^{\prime}+2 \omega_{0} \mu_{0} b+\left(\omega_{0} \sigma-\frac{\alpha_{1}}{2}\right) B=0
\end{aligned}
$$


Equation (30) and (31) admit solution of the form $(B, b) \propto(B, b) e^{\theta_{0} T_{1}}$ where $(B$ and $b)$ are constants then

$$
\begin{aligned}
\left(\frac{\alpha_{1}}{2}+\omega_{0} \sigma\right) b-2 \omega_{0}\left(\theta_{0}+\mu_{0}\right) B & =0 \\
2 \omega_{0}\left(\theta_{0}+\mu_{0}\right) b+\left(\omega_{0} \sigma-\frac{\alpha_{1}}{2}\right) B & =0
\end{aligned}
$$

the eigenvalues equation can be obtained as

$$
\theta_{0}=-\mu_{0} \pm \frac{\sqrt{\alpha_{1}^{2}-4 \omega_{0}^{2} \sigma^{2}}}{4 \omega_{0}}
$$

To analysis the nontrivial solution we followed steps similar to those in the preceding section, we obtains the following variational equations describing the stability of the steady state solutions:

$$
\begin{aligned}
& a_{1}^{\prime}=-\frac{2\left(\alpha_{1} \mu_{1}-3 \alpha_{3} \mu_{0}\right) a_{0}^{2}}{3 \alpha_{3} a_{0}^{2}+4 \alpha_{1}} a_{1}-\frac{1}{2} \sigma a_{0} \gamma_{1} \\
& \gamma_{1}^{\prime}=-\frac{6 \alpha_{3} \sigma a_{0}}{3 \alpha_{3} a_{0}^{2}+4 \alpha_{1}} a_{1}-\left(\frac{1}{2} \mu_{1} a_{0}^{2}+2 \mu_{0}\right) \gamma_{1}
\end{aligned}
$$

Equations (35) and (36) admit solution of the form $\left(a_{1}, \gamma_{1}\right) \propto\left(c_{1}, c_{2}\right) e^{\theta T_{1}}$ where $\left(c_{1}\right.$ and $\left.c_{2}\right)$ are constants, provided that

$$
\theta=-\frac{3 \alpha_{3} \mu_{1} a^{4}+8 \alpha_{1}\left(2 \mu_{0}+\mu_{1} a^{2}\right)}{4\left(4 \alpha_{1}+3 \alpha_{3} a^{2}\right)} \pm \frac{1}{4} \sqrt{\frac{256 \alpha_{1}^{2} \mu_{0}^{2}+96 \alpha_{1} \alpha_{3} a^{2}\left(2 \sigma^{2}+\mu_{0} G\right)+9 \alpha_{3}^{2} a^{4}\left(16 \sigma^{2}+G^{2}\right)}{\left(4 \alpha_{1}+3 \alpha_{3} a^{2}\right)^{2}}}
$$

so that $G=8 \mu_{0}+\mu_{1} a^{2}$

\section{Subharmonic resonance of order $1 / 4\left(\Omega \simeq 4 \omega_{0}\right)$}

In this case, $\Omega \simeq 4 \omega_{0}$. Then we can write

$$
\Omega=4 \omega_{0}+\epsilon \sigma
$$

and write

$$
\left(\Omega-3 \omega_{0}\right) T_{0}=\omega_{0} T_{0}+\epsilon \sigma T_{0}=\omega_{0} T_{0}+\sigma T_{1}
$$

Then eliminating the secular terms from the equation (8) yields

$$
2 i \omega_{0}\left(\mu_{1} A^{2} \bar{A}+A^{\prime}+\mu A\right)+\frac{1}{2} \alpha_{3} \bar{A}^{3} e^{i T_{1} \sigma}=0
$$

Using the polar form $A=\frac{1}{2} a\left(T_{1}\right) e^{i \beta\left(T_{1}\right)}$ into the equation (40) and separating real and imaginary parts, we obtain the following modulation equations:

$$
\begin{aligned}
& a^{\prime}=-\frac{\alpha_{3} a^{3} \sin (\gamma)}{16 \omega_{0}}-\frac{1}{4} \mu_{1} a^{3}-\mu_{0} a \\
& a \gamma^{\prime}=\sigma a-\frac{\alpha_{3} a^{3} \cos (\gamma)}{4 \omega_{0}}
\end{aligned}
$$

where $\gamma=\sigma T_{1}-4 \beta$.

For steady state solution, $a^{\prime}=\gamma^{\prime}=0$, in equations (41) and (42) we obtain

$$
\begin{aligned}
& \alpha_{3} a^{3} \sin (\gamma)=-4 \mu_{1} \omega_{0} a^{3}-16 \mu_{0} \omega_{0} a \\
& \alpha_{3} a^{3} \cos (\gamma)=4 \omega_{0} \sigma a
\end{aligned}
$$


Equations (43) and (44) show that there are two possibilities: (trivial solution ) at $a=0$ and (nontrivial solution) at $a \neq 0$. Squaring and adding (43) and (44) we get the frequency-response equation

$$
\sigma= \pm \frac{\sqrt{\alpha_{3}^{2} a^{4}-16 \omega_{0}^{2}\left(4 \mu_{0}+\mu_{1} a^{2}\right)^{2}}}{4 \omega_{0}}
$$

Now, the analysis of stability of the trivial solution is determined as in the preceding section as follows, we find that the trivial solution is stable for all values of $\sigma$ iff $\mu_{0} \geq 0$, by neglecting the non-linear terms in the equation (40) yields

$$
-2 i \omega_{0}\left(A^{\prime}+\mu_{0} A\right)=0
$$

to solve equation (46) one lets $A=e^{\frac{1}{2} i T_{1} \sigma}\left(B\left(T_{1}\right)+i b\left(T_{1}\right)\right)$ where B and b are real, separates real and imaginary parts and get

$$
\begin{aligned}
& b^{\prime}+\mu_{0} b+\frac{\sigma}{2} B=0 \\
& \frac{\sigma}{2} b-\mu_{0} B-B^{\prime}=0
\end{aligned}
$$

Equation (47) and (48) admit solution of the form $(B, b) \propto(B, b) e^{\theta_{0} T_{1}}$ where $(B, b)$ are constant then

$$
\begin{aligned}
& \left(\theta_{0}+\mu_{0}\right) b+\frac{\sigma}{2} B=0 \\
& \frac{\sigma}{2} b-\left(\theta_{0}+\mu_{0}\right) B=0
\end{aligned}
$$

the eigenvalues equation can be obtained as

$$
\theta_{0}=-\mu_{0} \mp \frac{i \sigma}{2}
$$

To analysis the nontrivial solution we followed steps similar to those in the preceding section, we obtains the following variational equations describing the stability of the steady state solutions:

$$
\begin{aligned}
& a_{1}^{\prime}=2 \mu_{0} a_{1}-\frac{\sigma a_{0}}{4} \gamma_{1} \\
& \gamma_{1}^{\prime}=-\left(\mu_{1} a_{0}^{2}+4 \mu_{0}\right) \gamma_{1}-\frac{2 \sigma}{a_{0}} a_{1}
\end{aligned}
$$

Equations (53) and (52) admit solution of the form $(B, b) \propto(B, b) e^{\theta T_{1}}$ where $(B, b)$ are constant, provided that

$$
\theta=\frac{-1}{2}\left(\mu_{1} a^{2}+2 \mu_{0}\right) \pm \frac{1}{2} \sqrt{\left(\mu_{1} a^{2}+6 \mu_{0}\right)^{2}+2 \sigma^{2}}
$$

\section{Numerical results and discussion}

This section presents numerical results in the form of frequency response diagrams obtained by solving the frequency response equations (16), (28) and (45) and stability conditions (20), (34), (37), (51) and (54). The numerical results are plotted in a groups of figures (1-7), (8-13), and (14-18), which represent the variation of the amplitude $(a)$ with the detuning parameter $(\sigma)$ for given values of the other parameters. In all figures, the soled lines represent stable solutions, while the dashed lines represent unstable solutions.

Figures (1-7) represent the frequency response curves of the harmonic resonance for the parameters $(F=$ $0.4, \omega_{0}=0.05, \mu_{0}=0.6, \mu_{1}=0.9, \alpha_{2}=0.1$ and $\alpha_{4}=0.2$ ). In (Fig.1) we have two curves the lower curve stable solutions and the upper one unstable solutions. These curves are symmetric about $\sigma=0$. When the coefficient of the external force $F$ is increased (decreased) the two curves move upward (downward) and have increased (decreased) magnitudes, the range of definition and stability does not change (Fig.2). If natural frequency $\omega_{0}$ is increased (decreased), we find that the two curves diverge(converge) from (to) each other, and have decreased (increased) magnitudes (Fig.3). If $\mu_{0}$ is increased (decreased), we find that the two curves diverge (converge) from (to) each other, and have decreased (increased) magnitudes for certain value of $\sigma$. By increasing $\sigma$ after this value, we note that their is no changes in the magnitudes (i.e. their exist a saturation) (Fig.4). If $\mu_{1}$ is increased (decreased), the 
two curves diverge (converge) from (to) each other, and have decreased (increased) magnitudes for certain value of $\sigma$. By increasing $\sigma$ after this value, we note that their is no changes in the magnitudes (i.e. their exist a saturation) (Fig.5). If $\alpha_{2}$ increased (decreased) we notice that the two curves move downward (upward) and have decreased (increased) magnitudes for certain value of $\sigma$. By increasing $\sigma$ after this value, we note that their is no changes in the magnitudes (i.e. their exist a saturation)(Fig.6). When $\alpha_{4}$ are increased (decreased) the two curves move downward (upward) and have decreased (increased) magnitudes for certain value of $\sigma$. By increasing $\sigma$ after this value, we note that their is no changes in the magnitudes (i.e. their exist a saturation) (Fig.7).

Figures (8-13) represent the frequency response curves of the subharmonic resonance of order $\frac{1}{2}$ for the parameters $\left(\omega_{0}=0.05, \mu_{0}=-2.0, \mu_{1}=2.0, \alpha_{1}=-0.12\right.$ and $\left.\alpha_{3}=-0.1\right)$. In (Fig.8) we note that the response amplitude has oval it is symmetric about $\sigma=0$ and it consist of two branches. The upper branch has stable solutions and the lower branch has unstable solutions, and there exist two saddle node bifurcations at the points $\sigma= \pm 6.359$. When the coefficient of nonlinear parametric excitation $\alpha_{1}$ decreases with negative values up to -0.5 the oval shifts upward and given semi closed curve, the regions of definition, multivalued and stability are increased and the saddle node bifurcations exist at the points $\sigma= \pm 12.07$. As $\alpha_{1}$ increases with negative values up to -0.01 the oval shrink and interfere, the zones of definition, multivalued and stability are decreased and the saddle node bifurcations exist at the points $\sigma= \pm 4.688$ as shown in (Fig.9). For increasing the value of coefficient of nonlinear parametric excitation $\alpha_{3}$ with negative values up to -0.05 the oval contracted respectively, the zones of definition, multivalued and stability are decreased and the saddle node bifurcations exist at the points $\sigma= \pm 2.905$. When the value of $\alpha_{3}$ decreases with the negative values up to -0.11 , we note that the oval expands and it has increased magnitudes, the zones of definition, multivalued and stability are increased and the saddle node bifurcations exist at the points $\sigma= \pm 7.974$. As $\alpha_{3}=-0.14$ we see that the multivalued solutions disappear, and the response amplitude given single valued solution which has unstable decreasing magnitudes as (Fig.10). When the damping factor $\mu_{0}$ decreases with negative values up to -3 , we note that the oval shifts upward and it has increased magnitudes respectively, the zones of definition, multivalued and stability are increased and the saddle node bifurcations exist at the points $\sigma= \pm 8.613$. As $\mu_{0}=-0.1$ the oval shifts downward and contracted and given semi closed curve, the regions of definition, multivalued and stability are decreased and the saddle node bifurcations exist at the points $\sigma= \pm 2.043$ as shown in (Fig.11). For decreasing the damping factor $\mu_{1}$ we note that the multivalued solutions disappear, and the response amplitude given single valued solution which has unstable decreasing magnitudes, for further decreasing of $\mu_{1}$ up to 1.5, we observe that the single valued curve move upward and has increasing unstable magnitudes, the zone of definition is increased for this decreasing of $\mu_{1}$, but, when $\mu_{1}$ take the values (4 and 8), we see that the oval is contracted and move downward respectively, the regions of definition, multivalued and stability are decreased, the saddle node bifurcations exist at the points $\sigma= \pm 2.924$ and $\sigma= \pm 1.981$ respectively as shown in (Fig.12). In (Fig.13), as $\omega_{0}$ increasing to take the values (0.07 and 0.09), the ovale is contracted respectively, the zones of definition, multivalued and stability are decreased, the saddle node bifurcations at the points $\sigma= \pm 3.55$ and $\sigma= \pm 2.564$ respectively. When $\omega_{0}=0.03$, the multivalued disappear and the response amplitude has single valued curve and it has unstable decreased magnitudes. For further decreasing of $\omega_{0}$, the single valued curve is separated into two branches.

Figures (14-18) represent the frequency response curves of the subharmonic resonance of order $\frac{1}{4}$ at almost the same parameters values taken at the preceding paragraph to represent the frequency response curves of the subharmonic resonance of order $\frac{1}{2}\left(\omega_{0}=0.05, \mu_{0}=-2.0, \mu_{1}=2.0\right.$ and $\left.\alpha_{3}=-0.1\right)$. In (Fig.14) the response amplitude $(a)$ has ellipse so that it symmetric about $\sigma=0$ and it consist of two branches. The upper branch has stable magnitudes and the lower branch has unstable solutions, and there exist two saddle node bifurcations at the points $\sigma= \pm 2.070$. As $\omega_{0}$ increasing to take the value 0.11 , the ellipse is shrank, the zones of definition, multivalued and stability are decreased, the saddle node bifurcations at the points $\sigma= \pm 0.910$. When $\omega_{0}$ decreasing up to $\omega_{0}=0.03$, the ellipse is expand, the zones of definition, multivalued and stability are increased, the saddle node bifurcations at the points $\sigma= \pm 3.672$, but, at the value of $\omega_{0}$ decreasing down to $\omega_{0}=0.01$, the multivalued disappear and the response amplitude has single valued curve and it has unstable decreased magnitudes as (Fig.15). When the value of coefficient of nonlinear parametric excitation $\alpha_{3}$ increaseing with the negative values up to -0.05 , we note that the ellipse contracted respectively, the zones of definition, multivalued and stability are decreased and the saddle node bifurcations exist at the points $\sigma= \pm 1.001$. and it has increased magnitudes, the zones of definition, multivalued and stability are increased and the saddle node bifurcations exist at the points $\sigma= \pm 11.01$. When the value of $\alpha_{3}$ decreases with the negative values up to -0.2, we note that the ellipse expands and it has increased magnitudes, the zones of definition, multivalued and stability are increased and the saddle node bifurcations exist at the points $\sigma= \pm 4.619$. As $\alpha_{3}=-0.4$ we see that the multivalued solution is disappear and the response amplitude given single valued curve which has unstable decreasing magnitudes as (Fig.16). When the damping factor $\mu_{0}$ decreases with negative values up to (-3.0 and -4.0$)$, we note that the ellipse shifts upward and it has 
increased magnitudes respectively, the zones of definition, multivalued and stability are increased and the saddle node bifurcations exist at the points $\sigma= \pm 3.101$ and $\sigma= \pm 4.137$ respectively. As $\mu_{0}$ increases with negative values up to (-1.0 and -0.5), the ellipse shifts downward and contracted, the regions of definition, multivalued and stability are decreased and the saddle node bifurcations exist at the points $\sigma= \pm 1.037$ and $\sigma= \pm 0.518$ respectively as shown in (Fig.17). For decreasing the damping factor $\mu_{1}$ we note that the multivalued solution is disappear and the response amplitude given single valued curve which has unstable decreasing magnitudes, for further decreasing of $\mu_{1}$ up to 0.5 , we observe that the single valued curve move upward and has increasing unstable magnitudes, the zone of definition is increased for this decreasing of $\mu_{1}$, but, when $\mu_{1}$ increasing to take the values ( 3 and 5 ), we see that the ellipse is contracted and move downward respectively, the regions of definition, multivalued and stability are decreased, the saddle node bifurcations exist at the points $\sigma= \pm 1.355$ and $\sigma= \pm 0.804$ respectively as shown in (Fig.18).

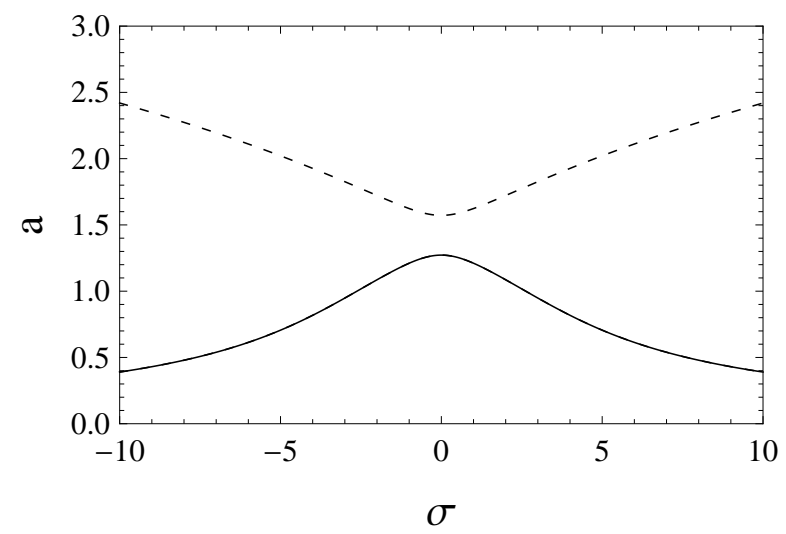

Fig.1: The frequency response curves of the harmonic resonance for the parameters $F=0.4, \omega_{0}=0.05$, $\mu_{0}=0.6, \mu_{1}=0.9, \alpha_{2}=0.1$ and $\alpha_{4}=0.2$

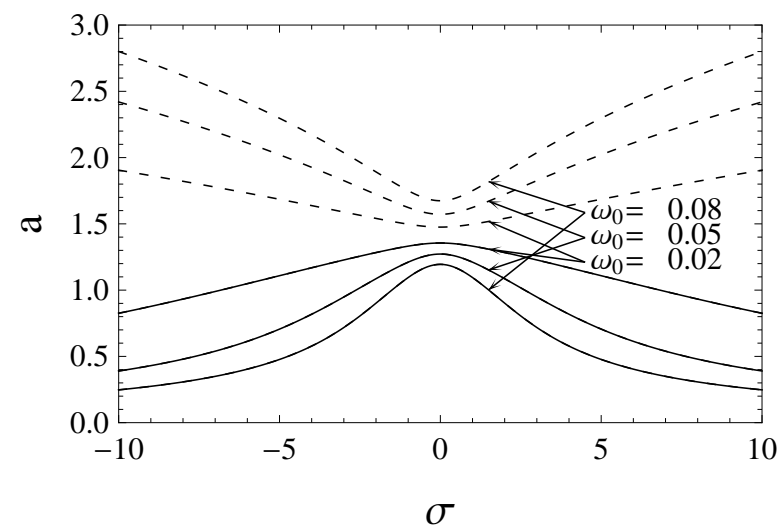

Fig.3: Variation of the amplitude of the response with the detuning parameter for increasing and decreasing $\omega_{0}$

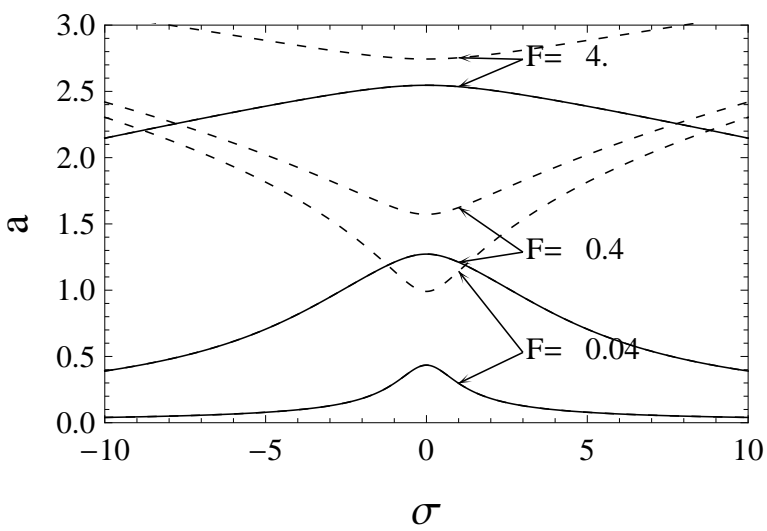

Fig.2: Variation of the amplitude of the response with the detuning parameter for increasing and decreasing $F$

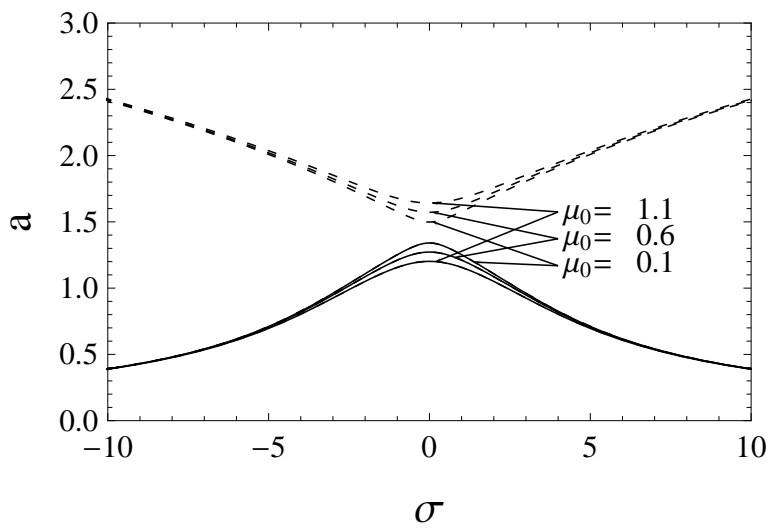

Fig.4: Variation of the amplitude of the response with the detuning parameter for increasing and decreasing $\mu_{0}$ 


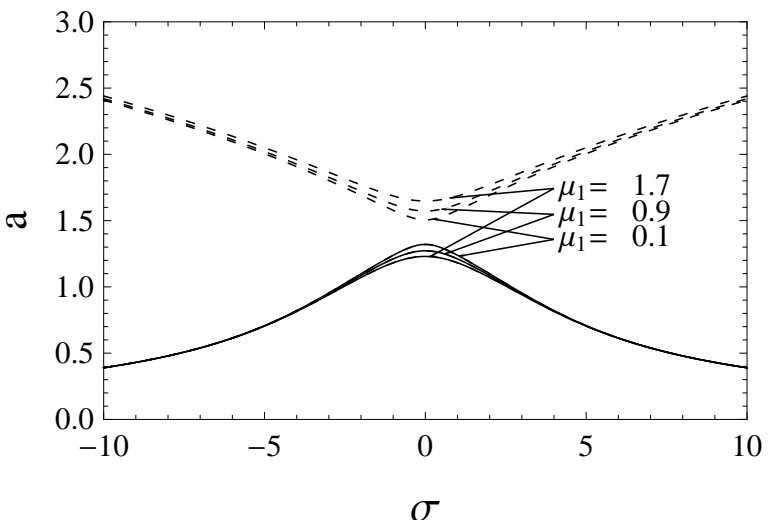

Fig.5: Variation of the amplitude of the response with the detuning parameter for increasing and decreasing $\mu_{1}$

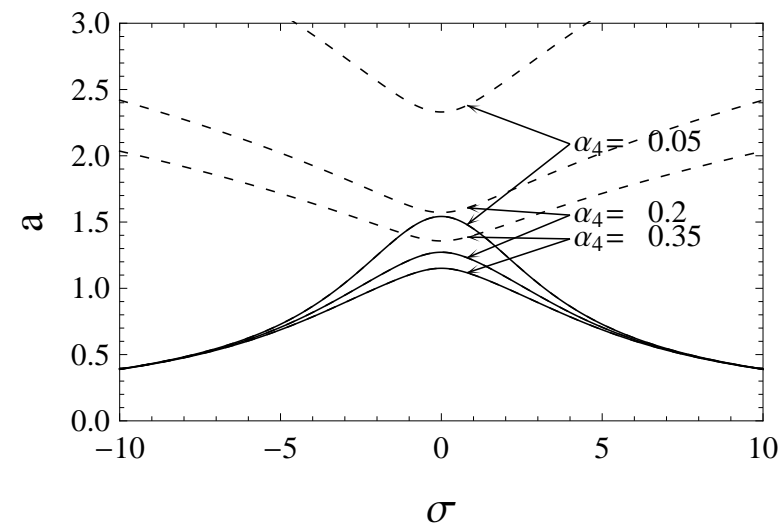

Fig.7:Variation of the amplitude of the response with the detuning parameter for increasing and decreasing $\alpha_{4}$

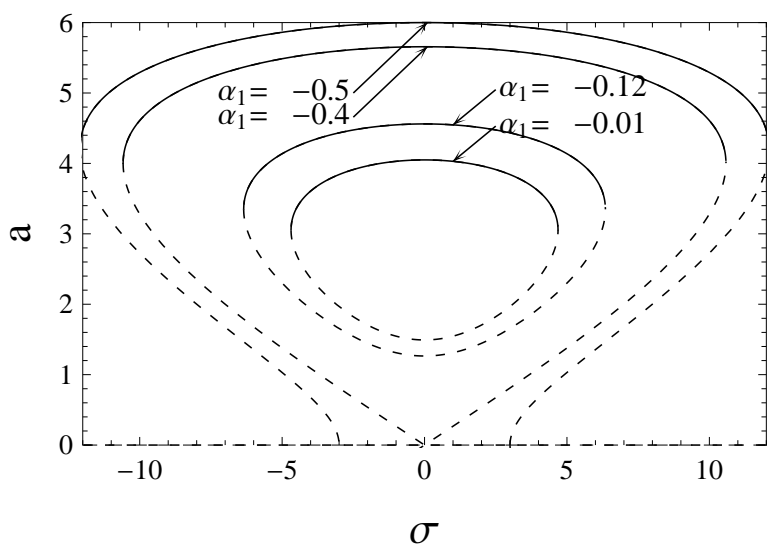

Fig.9: Variation of the amplitude of the response with the detuning parameter for increasing and decreasing $\alpha_{1}$

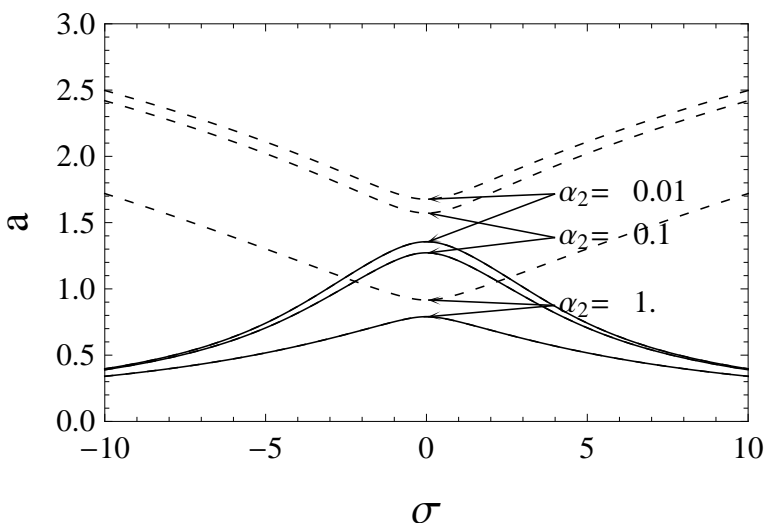

Fig.6: Variation of the amplitude of the response with the detuning parameter for increasing and decreasing $\alpha_{2}$

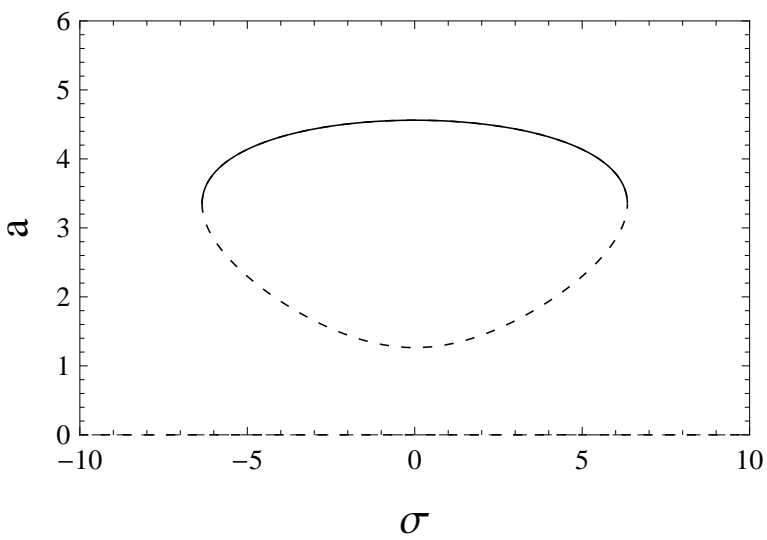

Fig.8: The frequency response curves of the subharmonic resonance of order $\frac{1}{2}$ for the parameters $\omega_{0}=0.05$ $\mu_{0}=-2.0, \mu_{1}=2.0, \alpha_{1}=-0.12$ and $\alpha_{3}=-0.1$

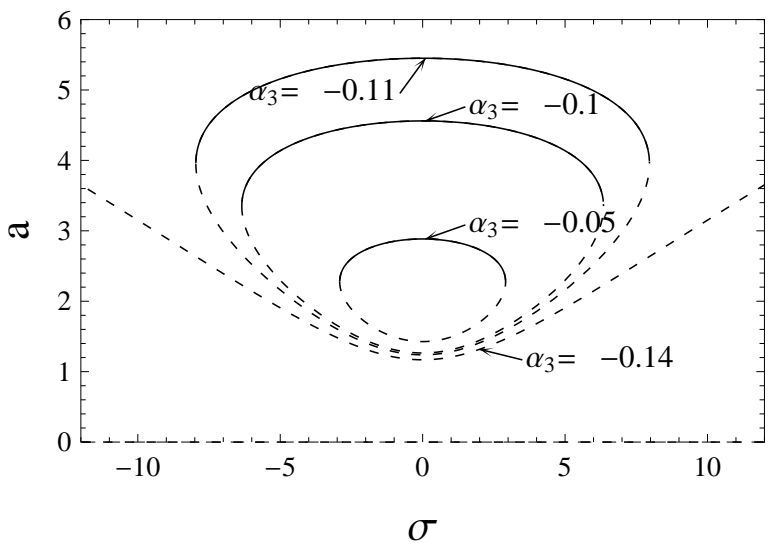

Fig.10: Variation of the amplitude of the response with the detuning parameter for increasing and decreasing $\alpha_{3}$ 


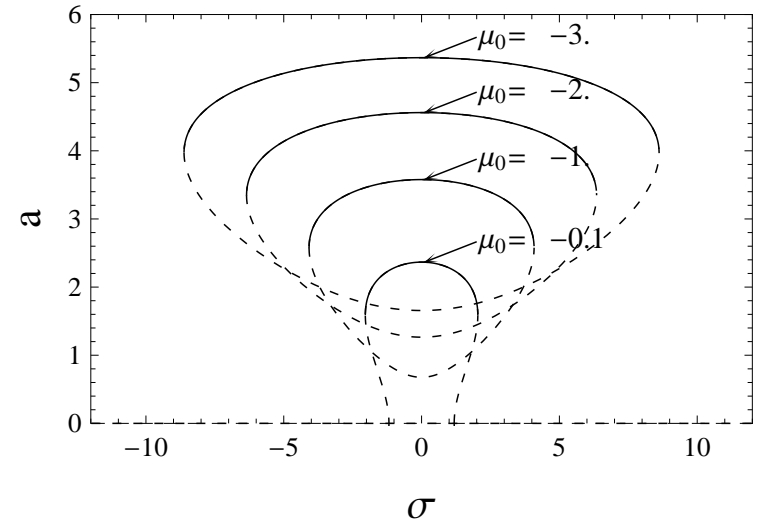

Fig.11:Variation of the amplitude of the response with the detuning parameter for increasing and decreasing $\mu_{0}$

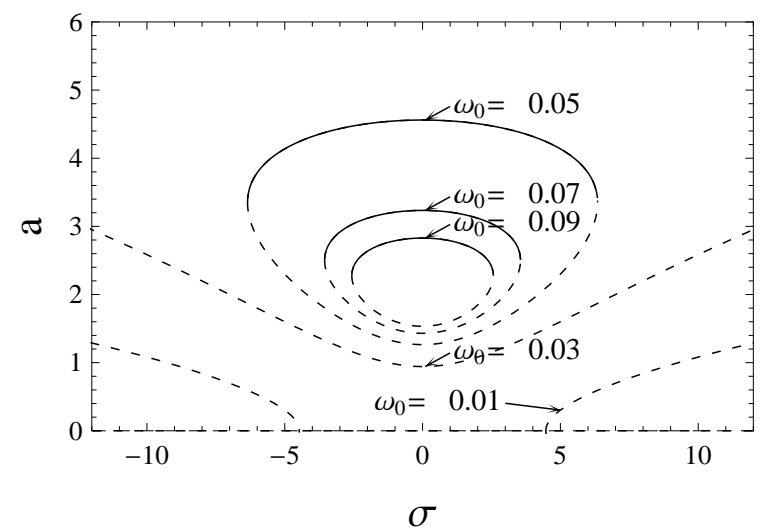

Fig.13: Variation of the amplitude of the response with the detuning parameter for increasing and decreasing $\omega_{0}$

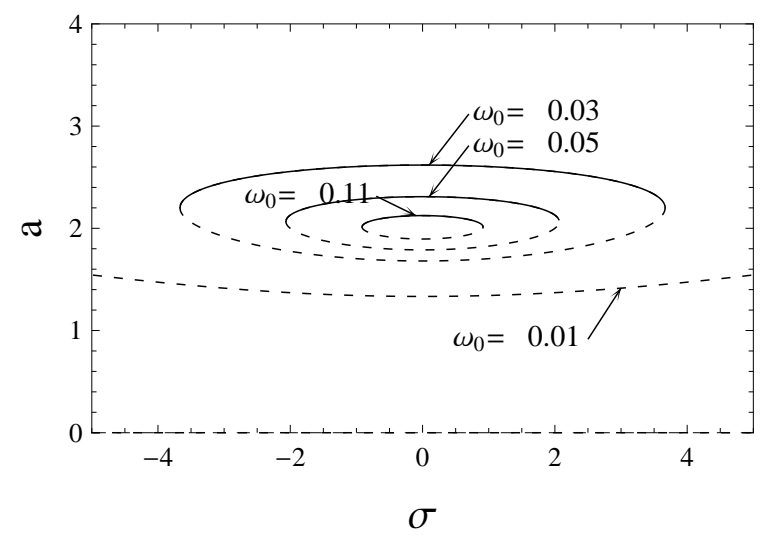

Fig.15:Variation of the amplitude of the response with the detuning parameter for increasing and decreasing $\omega_{0}$

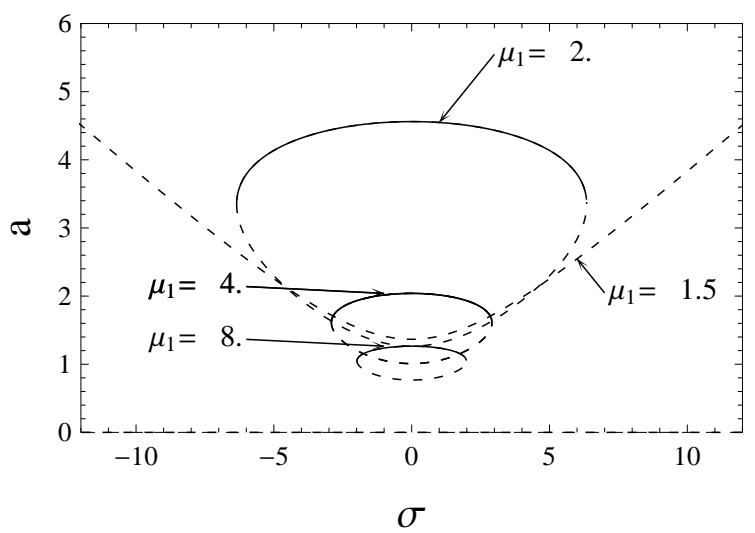

Fig.12: Variation of the amplitude of the response with the detuning parameter for increasing and decreasing $\mu_{1}$

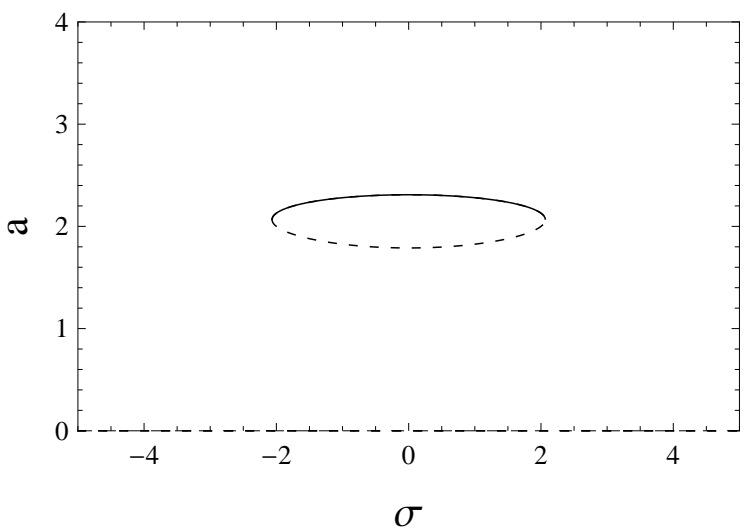

Fig.14: The frequency response curves of the subharmonic resonance of order $\frac{1}{4}$ for the parameters $\omega_{0}=0.05$ $\mu_{0}=-2.0, \mu_{1}=2.0$ and $\alpha_{3}=-0.1$

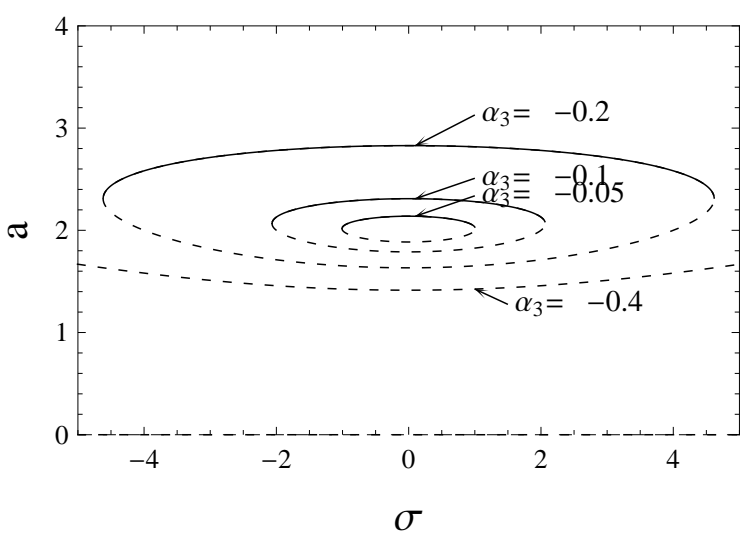

Fig.16: Variation of the amplitude of the response with the detuning parameter for increasing and decreasing $\alpha_{3}$ 


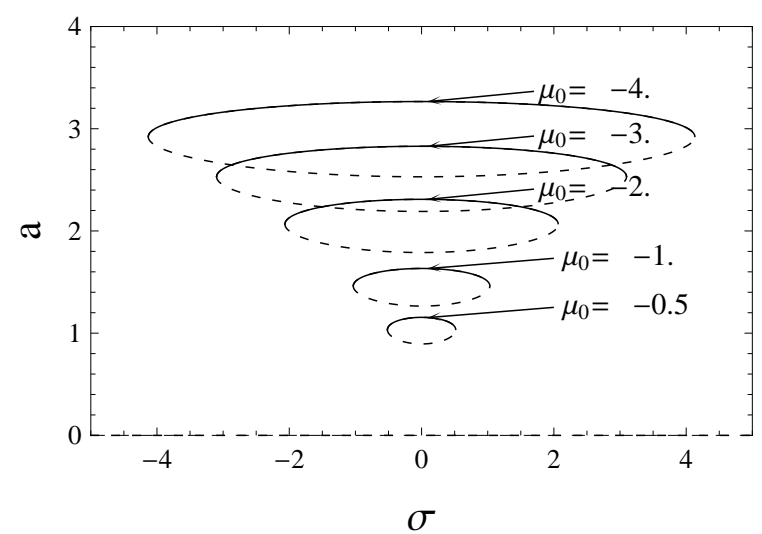

Fig.17: Variation of the amplitude of the response with the detuning parameter for increasing and decreasing $\mu_{0}$

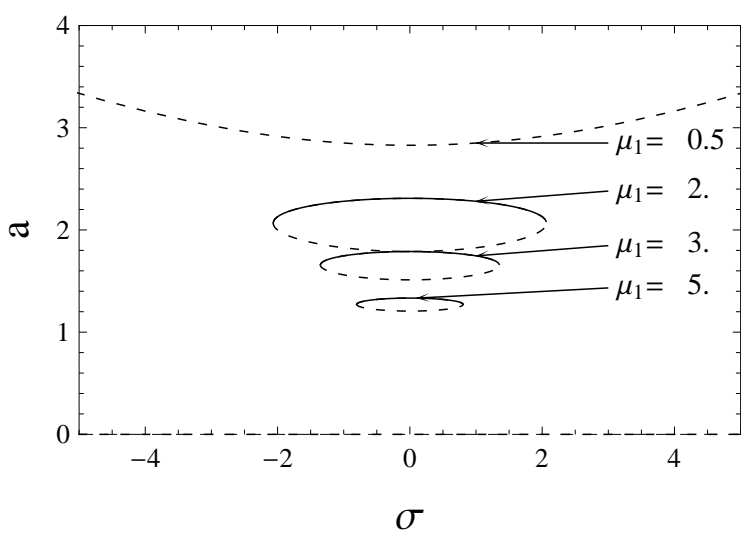

Fig.18: Variation of the amplitude of the response with the detuning parameter for increasing and decreasing $\mu_{1}$

\section{Conclusion}

In this paper we studied the weakly non-linear dynamic response (micro - electro - mechanical systems (MEMS)) which represented mathematical model a Van-der Pol equation subjected to a weakly non-linear parametric and forcing excitations. The study is devoted to harmonic resonance and subharmonic resonances of order (one - half and one - fourth). By using multiple scales method, for each resonance modulation equation, steady-state solutions, frequency response equation and stability of the steady-state solution are determined. Frequency response curves are plotted. Finally discussion for the figures are given.

From the frequency response curves of the harmonic resonance, we observe that the response amplitude consists of two curves so that the lower curve has stable solutions and the upper curve has unstable solutions. The two curves are symmetric a bout $\sigma=0$. The two curves move upward and downward when $F$ increased and decreased magnitudes respectively and the range of definition and stability does not change. When $\omega_{0}$ or $\mu_{0}$ or $\mu_{1}$ is increased the two curves diverge from each other, and have decreased magnitudes. When $\omega_{0}$ or $\mu_{0}$ or $\mu_{1}$ is decreased the two curves converge to each other, and have increased magnitudes. If $\alpha_{2}$ or $\alpha_{4}$ increased (decreased) we notice that the two curves move downward (upward) and have decreased (increased) magnitudes. We observe that their is a saturation phenomena at the cases of variations of $\mu_{0}, \mu_{1}, \alpha_{2}$ and $\alpha_{4}$.

From the frequency response curves of the subharmonic resonance of order $\frac{1}{2}$ we observe that the trivial solution has unstable solutions. The non trivial solution loses stability and the multivalued disappear when $\alpha_{3}=-0.14$, $\mu_{1}=1.5$ and $\omega_{0}=0.03$. The zones of definition are increased and decreased when the parameters $\alpha_{1}, \alpha_{3}, \mu_{0}$ and $\mu_{1}$ are decreased and increased respectively. The oval is divided into three branches when $\alpha_{1}=-0.5$ and $\mu_{0}=-0.1$ so that the lower branches are symmetric about $\sigma=0$ and have unstable solutions.

From the frequency response curves of the subharmonic resonance of order $\frac{1}{4}$ we observe that the ellipse contracted and expanded for increasing and decreasing $\omega_{0}$ and $\alpha_{3}$ respectively. The ellipse contracted (expanded) and move downward (upward) for increasing (decreasing) $\mu_{0}$ and $\mu_{1}$.

\section{References}

[1] L. R. Carley, J. A. Bain, G. K. Fedder, D.W. Greve, D. F. Guillou, M. S. C. Lu, T. Mukherjee, and S. Santhanam, Single-chip computers with microelectromechanical systems-based magnetic memory, J. Appl. Phys., 87, (2000), 66806685 .

[2] S. G. Adams, F. Bertsch, and N. C. MacDonald, Independent tuning of the linear and nonlinear stiffness coefficients of a micromechanical device, in IEEE Proc. MEMS, 96, (1996), 32-37.

[3] J. Bienstman, R. Puers, and J. Vandewalle, Periodic and chaotic behaviors of the autonomous impact resonator, in IEEE Proc. MEMS98, (1998), 562-567.

[4] Y. C. Wang, S. G. Adams, J. S. Thorp, N. C. MacDonald, P. Hartwell, and F. Bertsch, Chaos in MEMS, parameter estimation and its potential application, IEEE Trans. Circuits Syst. I: Fundam. Theory Appl., 45, (1998), 1013-1020. 
[5] N. A. Burnham, A. J. Kulik, G. Gremaud, and G. A. D. Briggs, Nanosubharmonics: The dynamics of small nonlinear contacts, Phys. Rev. Lett., 74, (1995), 5092-5095.

[6] A. F. El-Bassiouny and M. Eissa, Dynamics of a single-degree-of-freedom structure with quadratic, cubic and quartic nonlinearities to a harmonic resonance, Appl. Math. Comput., 139, (2003), 1-21.

[7] M. I. Younis, E. M. Abdel-Rahman, and A. H. Nayfeh, Dynamic simulations of a novel RF MEMS switch, in Proc. 7th Int. Conf. Modeling and Simulation of Microsystems: NanoTech, Boston, MA, (2004), 287-290.

[8] L. Lin, C. T.-C. Nguyen, R. T. Howe, and A. P. Pisano, Microelectromechanical filters for signal processing, in Proc. IEEE MEMS, Travemunde, Germany, (1992), 226-231.

[9] Z. Jin and Y. Wang, Electrostatic resonator with second superharmonic resonance, Sens. Actuators A, 64, (1998), 273-279.

[10] M. I. Younis and A. H. Nayfeh, A study of the nonlinear response of a resonant microbeam to electric actuation, Nonlinear Dyn., 31, (2003), 91-117.

[11] E. M. Abdel-Rahman and A. H. Nayfeh, Secondary resonances of electrically actuated resonant microsensors, Journal of Micromechanics and Microengineering, 13, (2003), 491-501.

[12] A. H. Nayfeh and M. I. Younis, Dynamics of MEMS resonators under superharmonic and subharmonic excitations, Journal of Micromechanics and Microengineering, 15, (2005), 1840-1847.

[13] N. Kacem, S. Hentz, D. Pinto, B. Reig, V. Nguyen, Nonlinear dynamics of nanomechanical beam resonators: improving the performance of NEMS-based sensors, Nanotechnology. 20, (2009), 275-501.

[14] F.M. Alsaleem, M.I. Younis, H.M. Ouakad, On the nonlinear resonances and dynamic pull-in of electrostatically actuated resonators, Journal of Micromechanics and Microengineering, 19, (2009),1-14.

[15] W. M. Zhang and G. Meng, Nonlinear Dynamic Analysis of Electrostatically Actuated Resonant MEMS Sensors Under Parametric Excitation, IEEE sensors journal, 7, no. 3, march (2007), 370-380.

[16] A. M. Elnaggar, Existence and Determination of Superharmonic Synchronizations as Solution,of a Quasi-Linear Physical System.Indian. J.Pure. Appl. Math., 16, (1985), 139-142.

[17] A. M. Elnaggar, A. F. El-Bassiouny and K. M. Khalil, Saddle-node bifurcation control for an odd non-linearity problem. Global J. of Pure and Applied Mathematics, 7, (2011), 213-229.

[18] W. M. Zhang, G. Meng and D. Chen, Stalility, Nonlinearity and Reliability of Electrostatically Actuated MEMS Devices, sensors journal, 7, (2007), 760-796.

[19] A. H. Nayfeh, Introduction to Perturbation Techniques, Wiley-Interscience, New York (1981). 